\title{
МАГНИТОИНДУЦИРОВАННЫЕ ЭФФЕКТЫ В ВИБРОННЫХ СТРУКТУРНЫХ ФАЗОВЫХ ПЕРЕХОДАХ
}

\author{
(Представил В. Хижняков)
}

Хорошо известны магнитные эффекты в таких системах с немагнитным фазовым переходом как сверхпроводники и экситонные изоляторы. Интерес к влиянию магнитного поля на структурные фазовые переходы возник в связи с разработкой вибронной теории $\left[{ }^{1-5}\right]$, на основе которой была предсказана возможность магнитного сдвига температуры Кюри сегнетоэлектрика $\left[{ }^{6,7}\right]$. Действие магнитного поля на сегнетоэлектрические характеристики изучалось экспериментально в ряде веществ ( $\left.\left.\mathrm{Pb}_{1-x} \mathrm{Ge}_{x} \mathrm{Te}, \mathrm{Pb}_{1-x} \mathrm{Sn}_{x} \mathrm{Te}, \mathrm{BaTiO}_{3}, \mathrm{SrTiO}_{3}, \mathrm{SbSJ}_{2} \mathrm{Sn}_{2} \mathrm{P}_{2} \mathrm{~S}_{6}\right){ }^{8-16}\right]$.

В полупроводниках-сегнетоэлектриках температура перехода поддается регулированию концентрацией носителей и во многих случаях оказывается низкой (десятки градусов). Иногда приходится иметь дело с виртуальными сегнетоэлектриками, у которых $T_{c}<0$.

Вибронная теория структурных фазовых переходов $\left[{ }^{1-5}\right]$ связывает электронную подсистему кристалла с сегнетоактивной частью фононной подсистемы. Поэтому внешнее воздействие, вызывающее изменения в электронной подсистеме, должно сказаться на свойствах сегнетоэлектрика. Магнитное поле действует на сегнетоэлектрические фазовые переходы из-за зеемановского расщепления и циклотронного квантования электронных спектров активных зон и вызывает, вообще говоря, перенормировку матричных элементов межзонного вибронного взаимодействия. Изменяется вклад ведущего межзонного электрон-фононного взаимодействия в частоту мягкой моды и, как следствие, происходит магнитоиндуцированный сдвиг температуры Кори. Другие характеристики сегнетоэлектрика зависят также от магнитного поля.

Направления магнитных сдвигов частоты мягкой моды и температуры Кюри фазового перехода второго рода $T_{C}$ сегнетоэлектрика-полупроводника определены в основном отношением между величинами спинового и циклотронного расщеплений активных электронных $30 н\left[{ }^{17}, 18\right]$. При $g_{n} \approx g_{p}$ ( $g_{n}$ и $g_{p}$ - спектроскопические факторы зоны прогодимости и валентной зоны) спиновое расщепление понижает $T_{C}$. В случае $g_{n} \neq g_{p}$ спиновое расщепление благоприятствует повышению $T_{C}$, если вклад от изменения порога для межзонных переходов в магнитоиндуцированный сдвиг частоты мягкой моды превалирует над вкладом от изменения чисел заполнения электронных состояний. Циклотронное квантование способствует понижению температуры Кюри, что было впервые показано И. Б. Берсукером с сотрудниками $\left[{ }^{19}\right]$. Магнитное поле может превратить виртуальный сегнетоэлектрический переход $\left(T_{C}<0\right)$ в реальный или привести қ исчезновению фазового перехода в системе. 
В случае достаточно низких температур тепловыми перебросами электронов из валентной зоны в зону проводимости можно пренебречь. Ширина разрешенных зон в полупроводниках обычно намного больше ширины запрещенной зоны. На основе перенормированной магнитным полем $(B)$ частоты мягкой моды в случае анизотропных параболических активных электронных зон аналогично $\left[{ }^{18,20}\right]$ получается следующая более общая в сравнении с $\left[{ }^{18,20}\right]$ (где использовалась менее гибкая аппроксимация электронного спектра) формула для сдвига точки Кюри $\left(\left|\delta_{n, p}^{(i)}\right|, \hbar \bar{\omega}_{\mathrm{c}}^{(i)} \ll \Delta\right):$

$$
\begin{aligned}
& \Delta T_{C}(B)=T_{C}(B)-T_{C}(0)= \\
& =\frac{3 \bar{V}^{2} \pi}{8\left(E_{0} \Delta\right)^{3 / 2} k_{B} \gamma_{a}\left(T_{C}(0)\right) \varphi} \sum_{i=1}^{\varphi}\left\{\left(\delta_{n}^{(i)}-\delta_{p}^{(i)}\right)^{2}-\frac{\left(\hbar \bar{\omega}_{c}^{(i)}\right)^{2}}{3}\right\} \text {, } \\
& \delta_{n, p}^{(i)}=\frac{1}{2} g_{n, p}^{(i)} \mu_{B} B ; \\
& \left|g_{n, p}^{(i)}\right|=\left[g_{n, p \|}^{2} \cos ^{2} \vartheta_{i}+g_{n, p \perp}^{2} \sin ^{2} \vartheta_{i}\right]^{1 / 2}, \\
& \bar{\hbar}_{c}^{(i)}=\mu_{B} B m_{0}\left[\left(m_{c m l}^{(i)}\right)^{-1}+\left(m_{c p}^{(i)}\right)^{-1}\right], \\
& m_{c n, p}^{(i)}=\left[\frac{m_{n, p \|} m_{n, p \perp}^{2}}{m_{n, p \perp} \sin ^{2} \vartheta_{i}+m_{n, p \|} \cos ^{2} \vartheta_{i}}\right]^{1 / 2} \text {, } \\
& \gamma_{a}(T)=\frac{1}{2\left(k_{B} T\right)^{2}} \sum_{\mathbf{q} j} A(\mathbf{q} j) \hbar \omega_{\mathbf{q} j} \operatorname{csch}^{2} \frac{\hbar \omega_{\mathbf{q} j}}{2 k_{B} T} \\
& A(\mathbf{q} j)=\frac{6 \hbar}{M_{j} \omega_{\mathbf{q} j}} B_{a}(01,01,-\mathbf{q} j, \mathbf{q} j) \\
& \bar{V}=V \sqrt{\frac{N}{N_{0}}}
\end{aligned}
$$

Здесь $\delta_{n, p}^{(i)}-$ спиновые расщепления зоны проводимости и валентной зоны; $g_{n, p}^{(i)}-$ соответствующие спектроскопические факторы; знаками $\|$ и $\perp$ обозначают продольные и поперечные компоненты; $\bar{\omega}_{c}^{(i)}-$ приведенная циклотронная частота; $m_{c n, p}^{(i)}-$ циклотронные массы электронов и дырок; $m_{0}-$ масса свободного электрона; $i=1, \ldots$, ф нумерует неэквивалентные долины в зоне Бриллюэна; $\vartheta_{i}-$ угол между В и осью вращения $i$-го изоэнергетического эллипсонда в $\mathbf{k}$-пространстве; $E_{0}$ - суммарная ширина разрешенных зон; $\Delta$ - затравочная ширина запрещенной зоны; $\omega_{q j}-$ фононные частоты; q - волновой вектор фононов, а $j$ - индекс колебательной ветви (для сегнетоактивного колебания $\boldsymbol{q}=0, j=1) ; B_{a}\left(\mathbf{q}_{1} j_{1}, \mathbf{q}_{2} j_{2}, \mathbf{q}_{3} j_{3}, \mathbf{q}_{4} j_{4}\right)$ - коэффициенты эффективной фононной ангармоничности четвертого порядка $\left[{ }^{1}\right] ; M_{j}-$ приведенная масса, отвечающая данной колебательной ветви; $A\left(\mathbf{q}_{j}\right)$ описывает фононную ангармоничность сегнетоэлектрического колебания; $V-$ константа межзонного электрон-фононного взаимодействия, $N$ - число электронов в активных зонах (валентная зона заполнена, а зона проводимости - пустая), $N_{0}$ - число элементарных ячеек кристалла. 
Из (1) видно, что спиновое расщепление $\delta_{n}^{(i)} \neq \delta_{p}^{(i)}$ ведет к повышению, а циклотронное квантование - к понижению температуры Кюри сегнетоэлектрического фазового перехода типа смещения. Это обусловлено тем, что спиновое расщепление с $g_{n}^{(i)} \neq g_{p}^{(i)}$ уменьшает, а циклотронное квантование - увеличивает эффективную щель между активными зонами.

Несобственные носители вызывают ослабление зависимости $T_{C}(B)$ и приводят в случае, когда число уровней Ландау под уровнем Ферми вырожденного полупроводника мало, к волнообразному характеру этой зависимости $\left[{ }^{18,20}\right]$. Теория позволяет объяснить наблюдаемое [ $\left.{ }^{9}\right]$ поведение температуры Кюри вырожденного сегнетоэлектрика-полупроводника $\mathrm{Pb}_{1-x} \mathrm{Ge}_{x} \mathrm{Te}$ во внешнем магнитном поле при различных концентрациях носителей. На рис. 1 приведены экспериментальные точки $\left[{ }^{9}\right]$ и теоретическая зависимость $\Delta T_{C}(B)$ для $\mathrm{Pb}_{0,99} \mathrm{Ge}_{0,01} \mathrm{Te}$ с концентрацией дырок $p=1,8 \cdot 10^{17} \mathrm{~cm}^{-3}\left(T_{C}(0) \approx 33 \mathrm{~K}\right)$, причем вклад в сдвиг точки Кюри, связанный с основными носителями, вычислен по (1). Вклад несобственных носителей в магнитное смещение $T_{C}$, вызывающее отклонение зависимости $\Delta T_{C}(B)$ от параболической, рассчитан аналогично $\left[{ }^{18,20}\right]$. Использованы следующие значения параметров: $\quad g_{\|}=60, g_{\perp}=17,5$, $m_{\perp}=0,022 m_{0}, m_{\|}=0,24 m_{0} \quad\left[{ }^{21}\right], \quad E_{0}=5$ эВ $\quad\left[{ }^{22}\right], \quad \Delta=0,2 \quad$ эВ $\left[{ }^{23}\right]$, $\gamma_{a}\left(T_{C}(0)\right)=0,2 \AA^{-2}, \bar{V}=0,9$ эВ $\AA^{-1}$ и учтено, что в соединениях типа $A^{\mathrm{IV}} B^{\mathrm{VI}} g_{n}^{(i)} \approx-g_{p}^{(i)}, m_{c n}^{(i)} \approx m_{c p}^{(i)}\left[{ }^{24-26}\right]$, а $\varphi=4$. Таким образом, повышение $T_{C}$ с ростом $B$ в вырожденных сегнетоэлектриках-полупроводниках $\mathrm{Pb}_{1-x} \mathrm{Ge}_{x} \mathrm{Te}\left[{ }^{8,9}\right]$ индуцировано спиновым расщеплением зон, превалирующим над действием циклотронного квантования. Полученное в $\left[{ }^{27,}{ }^{28}\right] \Delta T_{C}(B)>0$ приписано циклотронному расщеплению в гиперквантовом пределе.

Отметим, что в дальнейшем развитии теории влияния магнитного поля на сегнетоэлектрические свойства полупроводников типа $A^{\mathrm{IV}} B^{\mathrm{vI}}$ необходимо учесть в явном виде kр-взаимодействие * в электрон-фононном гамильтониане $\left[29,{ }^{30}\right]$ при $B \neq 0$, а также эффективный характер спина $\left[{ }^{31}\right]$ в базисных электронных состояниях.

Достаточно сильное магнитное поле может вызвать изменение рода структурного фазового перехода. Это обусловлено зависимостью коэффициента вибронного ангармонизма четвертого порядка в разложении свободной энергии системы по степеням параметра порядка от $B$. При этом спиновое расщепление $\delta_{n} \neq \delta_{p}$ благоприятствует фазовому переходу второго рода, а циклотронное квантование - фазовому переходу первого рода.

Магнитный сдвиг температуры сегнетоэлектрического фазового перехода первого рода $T_{0}$ не равняется сдвигу точки Кюри-Вейсса $T_{C}$ при наличии существенного вибронного ангармонизма или при сильной температурной зависимости $\gamma_{a}$. В случае, когда сегнетоэлектрик испытывает фазовый переход первого рода, должно наблюдаться влияние внешнего магнитного поля на температурный гистерезис перехода (магнитогистерезисный эффект). Возможные типы изменения температурной зависимости диэлектрической восприимчивости $\chi$ вблизи перехода первого рода представлены на рис. 2. Может индуцироваться смещение температурного интервала, в котором имеет место бифуркация равновесного состояния системы, к более высоким (рис. 2,a) или низким (рис. 2,б) темпера-

* kр-взаимодействие перенормирует константу межзонного вибронного взанмодействия $[29,30,9]$. Циклотронное квантование может в таком случае также дать положительный вклад в магнитный сдвиг $T_{c}\left[{ }^{9}\right]$. 


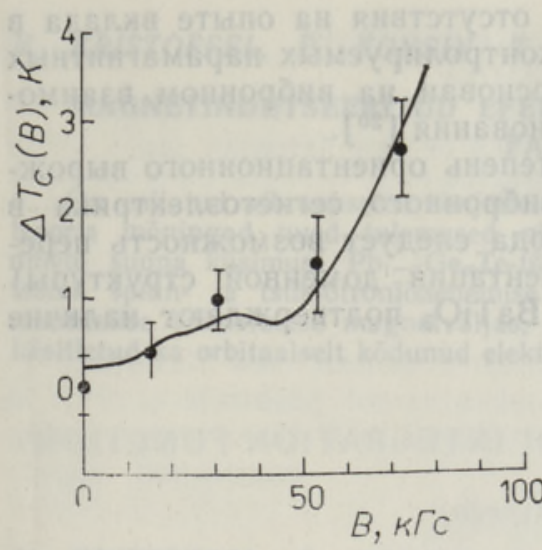

Рис. 1. Зависимость магнитоиндуцированного сдвига температуры Кюри $\mathrm{Pb}_{0,99} \mathrm{Ge}_{0,01} \mathrm{Te}$ от индукции магнитного поля при $p=1,8 \cdot 10^{17} \mathrm{~cm}^{-3}$. Точки - экспериментальные данные [9], сплошная линия - теоретические.

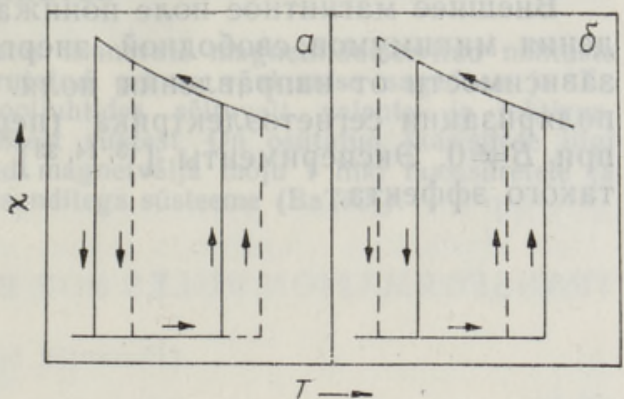

Рис. 2. Температурное поведение диэлектрической восприимчивости в случае сегнетоэлектрического фазового перехода первого рода. Сплошная линия $-B=0$, пунктирная $-B \neq 0$.

турам с одновременным его уширением или сужением. Конкретное поведение системы зависит от ее детальных характеристик.

Для рассмотрения влияния магнитного поля на сегнетоэлектрические фазовые переходы широкощельных диэлектриков типа $\mathrm{BaTiO}_{3}$ необходимо учесть орбитальную вырожденность активных электронных состояний ( $A_{1 g^{-}}$и $F_{1 u^{-}}$или $F_{1 u^{-}}$и $F_{2 g^{-}}$-состояния смешиваются $F_{1 u}$-колебанием) и магнитную перенормировку матричных элементов межзонного вибронного взаимодействия.

Трижды вырожденная в парафазе частота сегнетоэлектрического колебания $\left(F_{1 u}\right)$ расщепляется во внешнем магнитном поле $\mathbf{B} \|\langle 100\rangle$ на два компонента в согласии с симметрией $C_{4 h}$ высокосимметричной фазы $* *$. Высокотемпературная сегнетофаза имеет в поле симметрию $C_{4}$ или $C_{s}$ в зависимости от значений параметров активных электронных состояний. B $C_{s}$-фазе частота мягкой моды испытывает полное расщепление. Промежуточная по температуре сегнетофаза имеет симметрию $C_{s}$ или $C_{1}$, а низкотемпературная - симметрию $C_{1}$.

Нами получены формулы [ $\left.{ }^{32}\right]$, описывающие зависимости частот мягкой моды в парафазе и в высокотемпературной сегнетофазе, а также спонтанного искажения решетки от $B$. В пределе сильной связи мы нашли, что точка Кюри-Вейсса исследуемых систем повышается в магнитном поле. Для сегнетоэлектрика-диэлектрика $\mathrm{BaTiO}_{3}$ теоретическая величина сдвига $\Delta T_{0}(B) \approx \Delta T_{C}(B) \sim 10^{-3} \mathrm{~K}$ в поле $B=200 \mathrm{\kappa}$ с, что заметно меньше в сравнении с таковым для узкощельных полупроводников. Эксперименты указывают, действительно, на малые магнитные сдвиги температуры Кюри широкощельных сегнетоэлектриков $\left[{ }^{10,11}\right.$ $\left.{ }^{13-15}\right]$.

Не исключено, что в системах типа $\mathrm{BaTiO}_{3}$ является актуальным прямое влияние магнитного поля на динамику сегнетоактивной фононной подсистемы в результате действия силы Лоренца на движущиеся ионы.

** Расщепление частоты мягкой моды в парафазе вызвано влиянием магнитного поля на динамику решетки через ведущее межзонное электрон-фононное взаимодействие. 
Под вопросом остается также гарантия отсутствия на опыте вклада в магнитоиндуцированные эффекты от неконтролируемых парамагнитных примесей. Соответствующий механизм основан на вибронном взаимодействии примесных уровней с зонами основания $\left[{ }^{20}\right]$.

Внешнее магнитное поле понижает степень ориентационного вырождения минимумов свободной энергии вибронного сегнетоэлектрика в зависимости от направления поля. Отсюда следует возможность переполяризации сегнетоэлектрика (переориентация доменной структуры) при $B \neq 0$. Эксперименты [ $[13,14,33]$ для $\mathrm{BaTiO}_{3}$ подтверждают наличие такого эффекта.

\section{Л ИТЕРАТ УРА}

1. Kristoffel, N., Konsin, P. Ferroelectrics, 6, 3-12 (1973)

2. Konsin, P. Phys, status solidi (b), 76, № 2, 487-496 (1976).

3. Konsin, P. Phys, status solidi (b), 86, № 1, 57-66 (1978).

4. Bersuker, I. B., Vekhter, B. G. Ferroelectrics, 19, 137-150 (1978)

5. Kristoffel, $N$. N., Konsin, $P$. J. Ferroelectrics, 21, № 1-4, 477-479 (1978).

6. Консин П. Автореф. дис. канд. физ.-мат. наук. Тарту, 1970.

7. Консин П., Кристофель Н. Изв. АН ЭССР. Физ. Матем., 20, № 1, 37-47

8. Murase, K., Sugai, S., Takaoka, S., Katayama, S. In: Proc. 13th Int. Conf. Phys, Semicond. Rome, 1976, 305-308.

9. Takaoka, S., Murase, K. Phys. Rev. B, 20, № 7, 2823-2833 (1979)

10. Wagner, D., Bäuerle, D. Phys. Lett., 83A, № 7, 347-350 (1981).

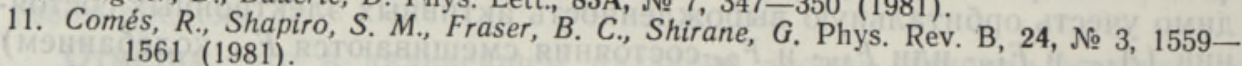

12. Brunel, L., C., Landwehr, G., Bussmann-Holder, A., Bilz, H., Balkanski, M., Massot, M., Ziolkiewicz, M. K. J. Phys., 42, № 12, C6-412-C6-414 (1981).

13. Флёрова С. А., Бочков О. Е. Письма в ЖЭТФ, 33, вып. 1, 37-40 (1981).

14. Флёрова С. А., Бочков О. Е. Кристаллография, 27, вып. 1, 198-201 (1982)

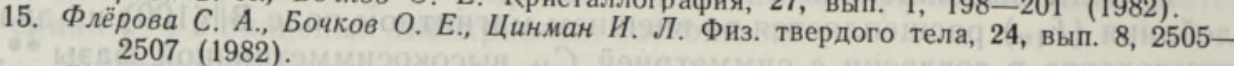

16. Flerova, S. A., Bochkov, O. E., Kudzin, A. Yu., Krochmal, Yu. D. Ferroelectrics, 45, 131-134 (1982).

17. Konsin, P., Ord, T. Phys. status solidi (b), 97, № 2, 609-615 (1980)

18. Konsin, P., Ord, T. Ferroelectrics, 45, $121-129$ (1982). 19. Вехтер Б. Г., Зенченко В. П., Берсукер И. Б. Физ. твердого тела, 18, вып. 8, 2325-
2330 (1976).

20. Konsin, P., Ord, T. In: Synergetics. Proc. Int. Symp. «Synergetics and Cooperative Phenomena in Solids and Macromolecules». Tallinn, «Valgus», 1983, 71-82.

21. Ichiguchi, T., Nishikawa, S., Murase, K. Solid State Commun., 34, № 5, 309-314 (1980)

22. Tung, Y. W., Cohen, M. L. Phys. Rev., 180, № 3, 823-826 (1969),

23. Цидильковский Н. М. Зонная структура полупроводннков. М., «Науказ, 1978.

24. Palik, E. D., Mitchell, D. L., Zemel, J. N. Phys. Rev., 135, № 3A, 763-778 (1964).

25. Lin, $P$. J.. Kleinman, L. Phys. Rev., 142, № 2, 478-489 (1966).

26. Gureev, D. M., Zasavitsky, I. I., Matsonashvili, B. N.. Shotov, A. P. In: Physics of Narrow Gap Semiconductors. Proc. 3rd Int. Conf. Warszawa, PWN-Polish Scientific Publishers, 1978, 109-114.

27. Волков В. Л., Литвинов В. И. Физ. твердого тела, 22, вып. 2. 617-619 (1980)

28. Volkov, V. L., Litvinov, V. I. Phys. Lett., 75A, № 5, 398-400 (1980).

29. Konsin, $P$. Ferroelectrics, 45, 45-50 (1982).

30. Консин П. И. Физ. твердого тела, 24, вып. 5, 1321-1327 (1982)

31. Mitchell, D. L., Wallis, R. F. Phys. Rev., 151, № 2, 581-595 (1966)

32. Konsin, P., Kristoffel, N., Ord, T. Solid State Commun., 49, № 4, 35i-355 (1984).

33. Новосильцев В. Н., Ролов Б. Н. Уч. зап. Латвийского университета, 195, 163168 (1973).

\section{Ннститут физики \\ Академии наук Эстонской ССР}


N. KRISTOFFEL, P. KONSIN, T. ORD

\section{MAGNETINDUTSEERITUD EFEKTID VIBROONSETES STRUKTUURSETES FAASISIIRETES}

On esitatud vibroonsetes senjettelektrikutes ilmnevate magnetindutseeritud nähtuste teooria mõningad uued tulemused ning arutatud pehme võnkumise sageduse ja $T_{c}$ nihete suuna küsimust $\mathrm{Pb}_{1-x} \mathrm{Ge}_{x} \mathrm{Te}$-tüüpi pooljuhtides sõltuvalt valents- ja juhtivustsooni spinn- ja tsüklotronlōhenemise vahelisest suhtest. On osutatud faasisiirde liigi muutumise võimalusele magnetväljas, uuritud magnetvälja mōju I liiki faasisiiretele ja käsitletud ka orbitaalselt kôdunud elektronseisunditega süsteeme $\left(\mathrm{BaTiO}_{3}\right)$.

\section{N. KRISTOFFEL, P. KONSIN, T. ORD}

\section{MAGNETIC-FIELD-INDUCED EFFECTS IN VIBRONIC STRUCTURAL PHASE TRANSITIONS}

Some new results on the theory of magnetic-field-induced effects in vibronic ferroelectrics are presented. The directions of soft-mode frequency and $T_{c}$ shifts for $\mathrm{Pb}_{1-x} \mathrm{Ge}_{x}$ Te-type semiconductors in dependence of the ratio of spin and cyclotron splittimgs of valence and conduction bands are discussed. The possibility of the change of phase transition order in magnetic field is suggested. The influence of the field on first-order transitions has been studied. The systems with orbitally degenerated active electronic states $\left(\mathrm{BaTiO}_{3}\right)$ are also considered. 